\title{
0 Doente Idoso no Serviço de Urgência
}

\author{
Older patients in Emergency Department
}

\author{
Pedro Oliveira, Beatriz Frutuoso, Rafaela Veríssimo, António Agripino \\ Centro Hospitar de Vila Nova de Gaia/Espinho
}

\section{Resumo}

Com o envelhecimento da população, prevê-se mais idosos no Serviço de Urgência (SU). Neste estudo pretendemos avaliar o tempo de estadia do Idoso num SU Polivalente, a sua triagem e orientação.

Realizamos um estudo retrospetivo, e selecionamos uma amostra aleatória simples correspondendo a $14 \%$ de todos os doentes admitidos durante um mês num SU Polivalente para adultos, sendo excluída a Urgência Obstétrica, correspondendo a 1252 dos 8872 episódios. Foram recolhidos dados demográficos, triagem de Manchester, especialidades envolvidas na avaliação do doente e a demora no SU.

0 tratamento estatístico foi elaborado usando SPSS $®$ versão 23, usando os testes de Qui-quadrado e os testes não paramétricos de Mann-Whitney e Kruskal-Wallis.

Nesta amostra, $53 \%$ dos doentes eram do sexo feminino, com idade média de 53.9 anos, sendo que $32.4 \%$ eram idosos ( $\geq 65$ anos). A mediana da demora no SU foi 215 minutos. As especialidades que observaram 0 maior número de doentes pela primeira vez foi a Medicina Geral e Familiar (MGF) em 30.2\%, seguida da Medicina Interna (25.3\%).

A mediana do tempo de estadia do idoso no SU foi superior ao grupo não idoso (271 vs 188 minutos) $(p<0.001)$. A atribuição de prioridade de maior severidade foi superior nos idosos $(p<0.001)$. Nos mais jovens, a especialidade mais atribuída pela triagem foi a MGF, nos idosos a especialidade era a Medicina Interna $(p<0.001)$. Os doentes idosos apresentaram maior probabilidade de serem internados $(p<0.001)$.

Assim, no doente idoso verificou-se uma maior demora no SU, uma triagem mais prioritária e a necessidade de cuidados mais diferenciados.

Palabras clave: Departamento de Emergencias. Geriatria. Duración de la estancia.

\section{Introdução}

Os idosos, indivíduos com 65 ou mais anos, são uma população em crescimento no Serviço de Urgência (SU)'. Estes doentes apresentam múltiplos problemas de saúde, consomem mais recursos que os doentes mais jovens e são mais frequentemente internados ${ }^{1,2}$. Apesar, do problema clínico resolvido a alta do doente está condicionada à sua funcionalidade, à necessidade de supervisão do tratamento e ao apoio social. A doença aguda em doentes idosos fragilizados agrava a sua deterioração e aumenta a mortalidade ${ }^{3}$.

Estes doentes frágeis beneficiam de capacidades dos profissionais de saúde específicas e modelos de organização adequados às suas necessidades complexas ${ }^{1}$.

A Triagem de Manchester, aplicada em diversos países, aplica um algoritmo em relação a sintomas e sinais na admissão do SU de forma a categorizar a prioridade de atendimento, entre os mais urgentes com atendimento imediato (Vermelho), 10 minutos (Laranja), 60 minutos (Amarela), 120 minutos (Verde) e os não urgentes em 240 minutos (Azul). Além da prioridade é atribuída a área/especialidade

\section{Abstract}

Aging is a problem affecting Emergency Medicine, requiring prepared sta$\mathrm{ff}$ for special needs of older patients. We intended to understand Colour Manchester Triage and length of stay (LOS) differences to younger patients and further orientation.

We conducted a retrospective, observational study, with a randomized sample of $14 \%$ of total patients admitted during a month in an Emergency department (1252 in 8872 episodes), excluding children and obstetric patients. We collected demographic data, Manchester triage, medical speciality involved, length of stay and destination. Statistical analysis was performed in SPSS $® 23.0$ using qui-square, Mann-Whitney and Kruskal-Wallis tests.

In our sample, 53\% were female, medium age was 53,9 years old (yo), and $32,4 \%$ were 65 yo or older. Median LOS in the Emergency Department (ED) was 215 minutes. Median LOS in geriatric patients were higher than younger population (271 vs 188 minutes) $(p<0.001)$. Priority triage was higher in older patients $(p<0.001)$.

Manchester triage attributed most patients to General Practice (30.2\%), followed by Internal Medicine (25.3\%). However, in geriatric population most patients were observed by Internal Medicine while younger patients were observed by General Practice $(p<0.001)$.

Older patients were more frequently admitted into ward than younger patients $(p<0.001)$.

Older patients presents higher LOS in the ED, require more immediate attention and required to be admitted in hospital ward more often. With ageing population ED will require to be adapted to their special needs. Keywords: Emergency Department. Geriatric Medicine. Lenght of stay.

que irá observar 0 doente ${ }^{4,5} . \mathrm{A}$ alguns doentes é atribuída a cor Branca, referindo-se a doentes transferidos de outros hospitais ou em situações excecionais.

Pretendemos avaliar a abordagem da triagem de Manchester em doentes idosos e não idosos, o tempo de permanência no Serviço de Urgência e o destino do doente.

\section{Material e métodos}

Utilizamos uma amostra aleatória simples, de 1252 num total de 8872 episódios de urgência, correspondendo a 14\% da população com mais de 17 anos, durante um mês, excluindo as admissões na Urgência Obstétrica e as reentradas no SU. Foram obtidos dados demográficos (idade, sexo), Triagem de Manchester (cor e especialidade de primeira observação), tempo de estadia no SU e destino após o episódio de urgência.

A idade foi dicotomizada em grupos etários com idades $<65$ $e \geq 65$ anos. A abordagem por especialidade foi estratificada em Medicina Geral e Familiar (MGF), Medicina Interna (MI), Cirurgia Geral (CG), Médico-Cirúrgica - Oftalmologia e Oto- 
Gráfico 1. Distribuição pelas especialidades (MC - Médico-Cirúrgicas; SEM - Subespecialidades médicas; SEC - Subespecialidades cirúrgicas; MI - Medicina Interna; CG - Cirurgia Geral; ORT - Ortopedia; MGF - Medicina Geral e Familiar; TT - População total)

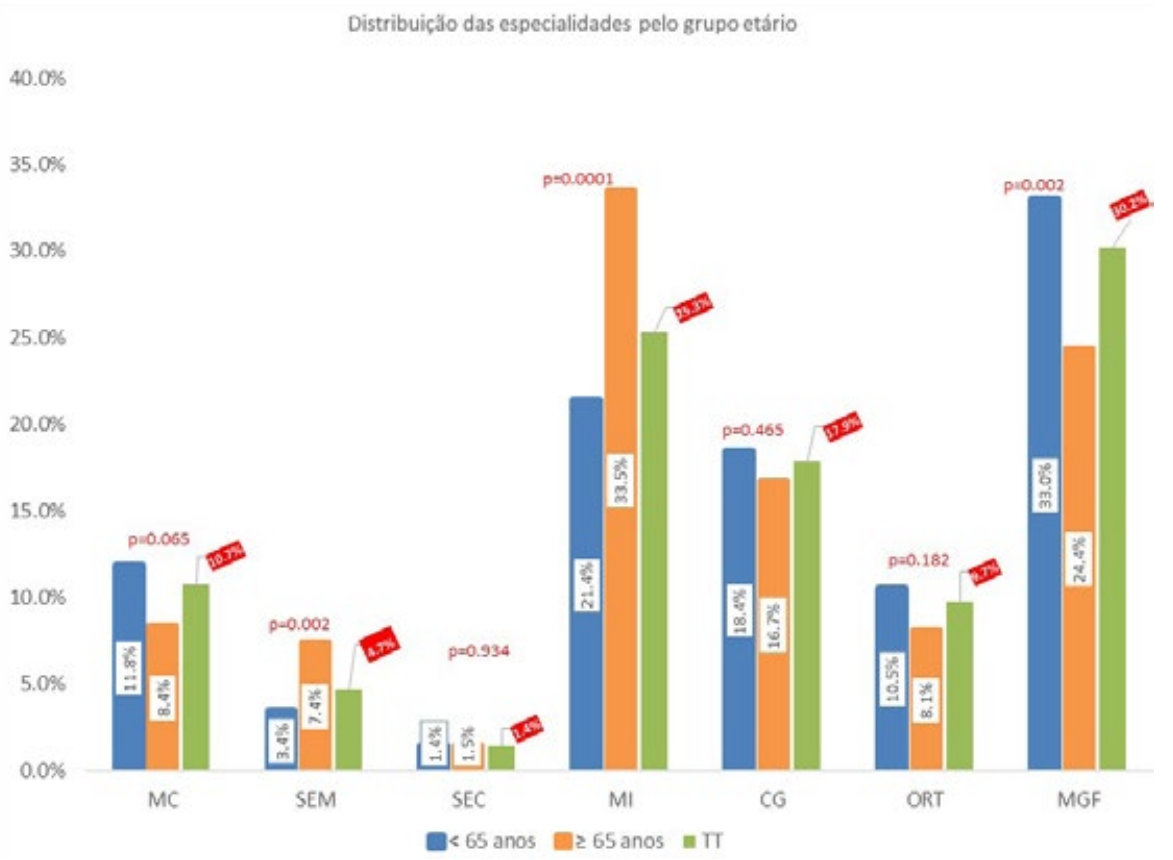

Tabela 1. Comparação da amostra entre grupos por sexo, tempo de estadia, triagem e destino

\begin{tabular}{|l|c|c|c|c|}
\hline $\begin{array}{l}\text { Características } \\
\text { da amostra }\end{array}$ & Total & $<65$ anos & $\geq 65$ anos & Valor $p$ \\
\hline Número & 1252 & $846(67.6 \%)$ & $406(32.4 \%)$ & \\
\hline Sexo (feminino) & $663(53 \%)$ & $439(51.9 \%)$ & $224(55.2 \%)$ & $p=0.304$ \\
\hline $\begin{array}{l}\text { Tempo no SU } \\
\text { (mediana) (minutos) }\end{array}$ & 215 & 188 & 271 & $\mathrm{p}=0.0001$ \\
\hline Triagem & $6(0.5 \%)$ & $4(0.5 \%)$ & $2(0.5 \%)$ & $\mathrm{p}=0.962$ \\
\hline Vermelho & $146(11.7 \%)$ & $72(8.5 \%)$ & $74(18.2 \%)$ & $\mathrm{p}=0.0001$ \\
\hline Laranja & $742(59.3 \%)$ & $509(60.2)$ & $233(57.4 \%)$ & $\mathrm{p}=0.349$ \\
\hline Amarelo & $298(23.8 \%)$ & $224(26.5 \%)$ & $74(18.2 \%)$ & $\mathrm{p}=0.001$ \\
\hline Verde & $2(0.2 \%)$ & $1(0.1 \%)$ & $1(0.2 \%)$ & $\mathrm{p}=0.544$ \\
\hline Azul & $58(4.5 \%)$ & $36(4.2 \%)$ & $22(5.4 \%)$ & $\mathrm{p}=0.389$ \\
\hline Branco & \multicolumn{5}{|l|}{} \\
\hline Destino & $124(9.9 \%)$ & $50(5.9 \%)$ & $74(18.2 \%)$ & $\mathrm{p}=0.0001$ \\
\hline Internamento & $106(8.5 \%)$ & $71(8.4 \%)$ & $35(8.6 \%)$ & $\mathrm{p}=0.978$ \\
\hline Consulta Externa & $959(76.6 \%)$ & $674(79.7 \%)$ & $285(70.2 \%)$ & $\mathrm{p}=0.0001$ \\
\hline Domicílio & $1(0.1 \%)$ & $0(0 \%)$ & $1(0.2 \%)$ & $\mathrm{p}=0.3240 \mathrm{NS}$ \\
\hline Óbito & $23(2.2 \%)$ & $19(2.2 \%)$ & $4(1.0 \%)$ & $\mathrm{p}=0.176$ \\
\hline Transferido & $39(3.1 \%)$ & $32(3.8 \%)$ & $7(1.7 \%)$ & $\mathrm{p}=0.056$ \\
\hline Abandono &
\end{tabular}

rrinolaringologia - (MC), Ortopedia (ORT), Subespecialidades médicas (SEM) e Subespecialidades cirúrgicas (SEC).

A triagem de Manchester permite identificar a gravidade dos doentes, sendo que os da categoria Vermelho são os mais graves, seguidos do Laranja, Amarelo, Verde e Azul.

0 tratamento estatístico foi elaborado usando SPSS $®$ versão 23, usando os testes de Qui-quadrado e os testes não paramétricos de Mann-Whitney e Kruskal-Wallis para comparação de médias e medianas para valor $p<0.05$.

\section{Resultados}

Nesta amostra, 53\% corresponderam a doentes do sexo feminino, não havendo diferença significativa entre os grupos, e apresentavam idade média de 53.9 anos, sendo que $32.4 \%$ eram idosos ( $\geq 65$ anos). A mediana da demora no SU foi de 215 minutos. A prioridade mais frequentemente atribuída foi a cor amarela (59.3\%), seguido da verde (23.8\%) (Tabela 1). A especialidade a observar 0 doente pela primeira vez, mais frequentemente, foi a MGF (30.2\%) seguida da MI (25.3\%). A triagem atribui maior percentagem à MGF $(33 \%, p=0.002)$ nos doentes com menos de 65 anos enquanto nos idosos a maior percentagem foi atribuída à Medicina Interna (33,5\%, $p=0.001$ ) (Gráfico 1).

Verificámos uma diferença na atribuição de prioridades na triagem, sendo com maior severidade (Laranja, $p=0.001$ ) nos idosos enquanto no grupo jovem se verificou menor gravidade (Verde, $p=0.001$ ) (Tabela1). Da análise efetuada verificamos que a mediana do tempo de estadia do idoso no SU era superior (271 vs 188 minutos) ( $p=0.001$ ) sem diferenças para o sexo, sendo que essa diferença foi signifi- 


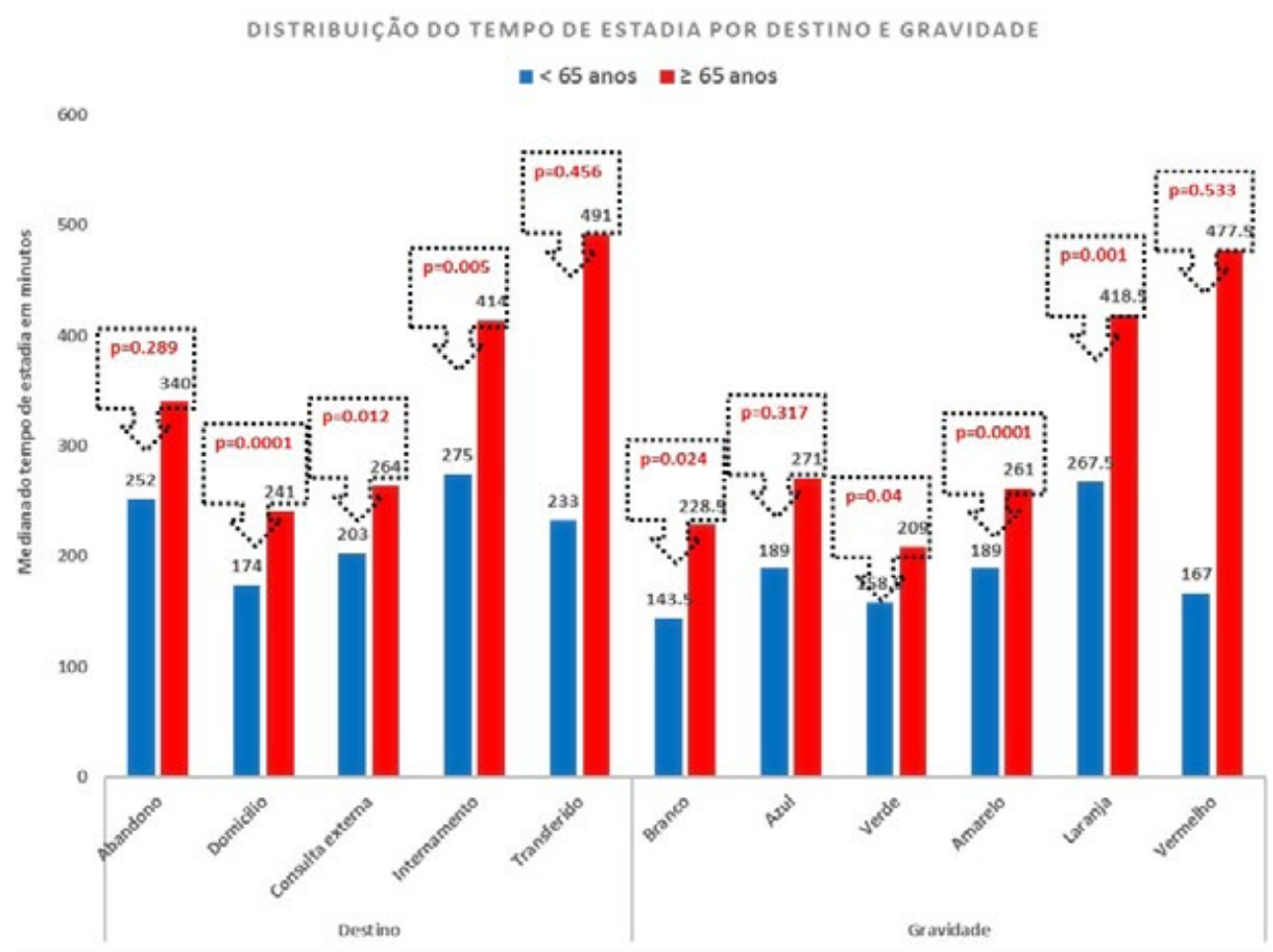

cativa para os graus de prioridade Verde, Amarela e Laranja (Gráfico 2).

Identificamos que existe uma relação significativa entre 0 destino e a triagem Manchester, sendo que os doentes com triagem mais grave eram mais frequentemente internados, independentemente do grupo etário (tabela 2; $p=0.001$ ) e na análise comparativa de grupos objetivamos que os doen- tes idosos apresentavam uma maior probabilidade de serem internados em relação aos não idosos (Idosos: 74/406 (18.2\%) e Não idosos 50/846 (5.9\%)) (Tabela1; $p=0.0001$ ). A mediana do tempo de estadia foi mais longo para o grupo $\geq 65$ anos ( $p=0.0001$; Tabela 1) e que se traduz no destino relativo ao internamento $(p=0.005)$, consulta externa $(\mathrm{p}=0.012)$ e domicílio ( $\mathrm{p=0.0001)}$ (Gráfico 2).

Tabela 2. Distribuição do Destino segundo Triagem Manchester

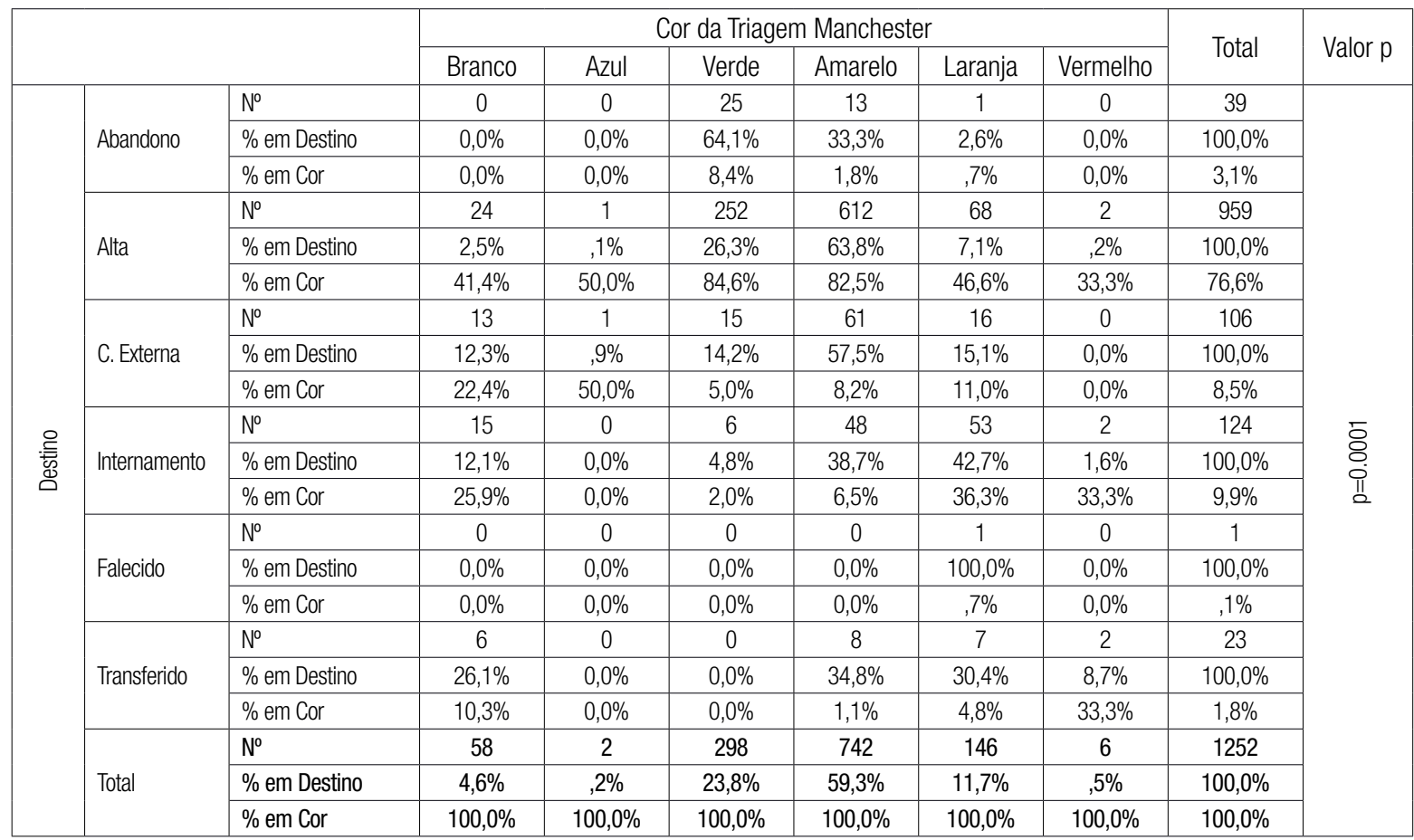




\section{Discussão}

O serviço de urgência polivalente de adultos do Centro Hospitalar Gaia-Espinho incorpora as diferentes especialidades para 0 atendimento ao doente urgente e emergente. A atribuição de prioridade é realizada por enfermeiros que aplicam o sistema de triagem de Manchester e segundo os fluxogramas instituídos enviam para as diferentes especialidades.

A atribuição de prioridade de maior severidade aos doentes idosos vai de encontro ao que se encontra publicado, porque, devido às comorbilidades e à fragilidade associada a estes doentes, necessitando de atendimento prioritário $0^{1,2}$. Assim, a maior gravidade orienta os doentes para uma especialidade vocacionada para as situações mais complexas, de forma a dar resposta às necessidades destes doentes. Apesar de não ter sido abordado o diagnóstico e as comorbilidades, é de esperar um maior número de doenças crónicas nesta população, condicionando a necessidade de maiores cuidados para estabilização das suas patologias ${ }^{1}$. 0 nosso estudo demonstrou esta necessidade verificando-se que a maioria dos idosos foram observados pelas especialidades básicas hospitalares, com predomínio da Medicina Interna em $1 / 3$ e a MGF em apenas $1 / 4$ dos casos. Nas restantes áreas clínicas é de referir a importância das médico-cirúrgicas em $10 \%$ dos casos e a necessidade residual das subespecialidades médicas ou cirúrgicas.

A triagem como sistema de prioridades indica um atendimento mais rápido para os doentes mais graves, contudo verificamos que estes doentes apresentaram maior tempo de permanência no SU. Isto poderá explicar-se devido à complexidade diagnóstica e à necessidade de tratamentos mais complexos de forma a estabilizar o doente para a alta clínica do SU (domicilio ou internamento). Pensamos que a diferença, no tempo de estadia, entre os idosos e os mais jovens com a mesma gravidade deve-se ainda à dificuldade da obtenção da semiologia e exame objetivo associado ao número de comorbilidades, condicionando o diagnóstico diferencial para além de uma resposta mais demorada ao tratamento a instituir, atendendo à maior probabilidade de efeitos adversos da medicação nesta população?

Contudo, este trabalho mostrou que os doentes com prioridade mais baixa têm alta do SU antes dos doentes com prioridade superior. Isto pode-se dever por não apresentarem situações clínicas complexas e necessidade de investigação complementar.

Verificamos que o sexo não influencia a atribuição de gravidade nos doentes idosos e que, apesar de uma ligeira maioria de doentes do sexo feminino, esta não foi significativa.

A triagem de Manchester mostrou-se eficaz em detetar os doentes com maior probabilidade de necessitarem de observação mais precoce e posterior admissão na enfermaria ${ }^{4}$.
De acordo com a literatura, os doentes mais idosos apresentaram uma maior taxa de internamento, podendo isto estar relacionado com a gravidade das doenças e a maior fragilidade, necessitando de um nível de cuidados diferenciados, apenas disponível em modelo de internamento ${ }^{1,2}$.

0 nosso estudo tem várias limitações. Primeiro, estudamos dados retrospetivos e, portanto, o número de preditores disponíveis e resultados relacionados eram limitados. Idealmente, os preditores, tais como cognição, estado funcional e readmissões deveriam ser usados, mas estes não estavam disponíveis neste estudo retrospetivo. Em segundo lugar, 0 fato de que nós encontramos alguns resultados inesperados, por exemplo tempo de estadia superior no grupo idoso com gravidade semelhante relativamente ao grupo jovem, salienta ainda a necessidade de validação externa, uma vez que é impossível distinguir se estes resultados são específicos para nossa coorte, resultado ocasional ou reprodutível em outras coortes.

Os pontos fortes do presente estudo são que a predição do Sistema de Triagem de Manchester pode ser aplicado ao doente idoso. Isso pode melhorar uma futura adaptação da doença crónica a este tipo de triagem. Outro ponto forte é a orientação para as especialidades básicas hospitalares que garantem e asseguram 0 desenvolvimento de intervenções aos doentes com alto risco de um evento negativo. Tais orientações são de importância no cenário medicina de emergência, permitindo que os médicos em serviço de urgência tomar decisões informadas sobre as estratégias de diagnóstico e terapêutica em doentes idosos e a implementação de medidas para evitar mau resultado.

\section{Conclusão}

No nosso Centro Hospitalar, o doente idoso apresenta maior tempo de permanência no SU, uma triagem mais prioritária, é observado preferencialmente por Medicina Interna e é mais internado que o doente mais jovem. Isto reflete a necessidade de cuidados diferenciados para os doentes idosos 0 envelhecimento da população levará à necessidade de restruturar os serviços de urgência para se prepararem para esta realidade crescente.

\section{Bibliografia}

1. Salvi F, Morichi V, Grilli A et al. The Elderly in the Emergency Department: A Critical Review of Problems and Solutions. Intern Emerg Med 2007;2:292-301.

2. Hominick K, McLeod V, Rockwood K. Characteristics of Older Adults Admitted to Hospital versus Those Discharged Home, in Emergency Department patients Referred to Internal Medicine, Canad Geriatrics J 2016; 19(1):9-14.

3. Gelder J, Lucke JÁ, Heim N et al. Predicting Mortality in acutely Hospitalized older patients: a retrospective cohort study, Intern Emerg Med 2016 11:587-594.

4. Cooke MW, Jinks S, Does de Manchester Triage System detect the critically ill?, J Accid Emerg Med 1999;16:179-181.

5. Guedes HM, Martins JC, Chianca TC. Valor de Predição do Sistema de Triagem de Tabela 2. Distribuição do Destino segundo Triagem Manchester: avaliação dos desfechos clínicos de pacientes. Rev Bras Enferm 2015; 68(1): 45-51. 